\title{
Comparison of vaginal misoprostol, laminaria, and isosorbide dinitrate on cervical preparation and labor duration of term parturient: a randomized double-blind clinical trial
}

\author{
Behnaz Souizi ${ }^{1}$, Forough Mortazavi ${ }^{2}$, Sima Haeri $^{3}$, Fateme Borzoee ${ }^{4}$
}

${ }^{1}$ MD., Gynecologist, Assistant Professor, Department of Obstetrics and Gynecology, School of Medicine, Sabzevar University of Medical Sciences, Sabzevar, Iran

${ }^{2}$ Ph.D. of Reproductive Health, Assistant Professor, Department of Midwifery, School of Medicine, Sabzevar University of Medical Sciences, Sabzevar, Iran

${ }^{3}$ Candidate of Medicine, School of Medicine, Sabzevar University of Medical Sciences, Sabzevar, Iran

${ }^{4}$ M.Sc. of Nursing, Instructor, Department of Operating Room, School of Paramedics, Sabzevar University of Medical Sciences, Sabzevar, Iran

\section{Type of article: Original}

\begin{abstract}
Background: Cervical ripening plays an important role in successful labor induction.

Objective: This study aimed to compare the effects of misoprostol, laminaria tent, and isosorbide dinitrate (ISDN) on cervical ripening.

Methods: This double-blind three-armed clinical trial was conducted at Shahidan Mombini Teaching Hospital in Sabzevar, Iran, in 2016 on 96 singleton term pregnant women. Participants were randomly allocated to receive either two 20-mg ISDN tablets vaginally every 4 hours for a maximum of three doses or $25 \mathrm{mcg}$ misoprostol vaginally every 6 hours for a maximum of two doses or laminaria tent for a maximum of 12 hours. The method of randomization was covariate adaptive randomization and the primary outcome measures were Bishop Score changes and labor duration. SPSS software version 18 was used for statistical analyses. Kruskal-Wallis, chisquare and ANOVA tests were applied for data analyses.

Results: Bishop Score changes were higher in the misoprostol group than in the two other groups $(\mathrm{p}=0.014)$. Time from start of medication to active phase of labor and delivery were $6.22 \pm 3.11$ and $11.78 \pm 5.3$ minutes in the misoprostol group, $11.25 \pm 3.07$ and $17.62 \pm 4.07$ minutes in the laminaria group, and $10.12 \pm 3.48$ and $17.37 \pm 4.79$ minutes in the ISDN group respectively $(\mathrm{p}<0.001)$. Cesarean rate was higher in the misoprostol group than the two other groups $(\mathrm{p}=0.016)$. No significant differences were observed between the study groups in terms of Apgar score and meconium-stained amniotic fluid.

Conclusions: Use of misoprostol results in more improvement of Bishop Score and reduced length of labor phases in comparison to laminaria tent and ISDN.

Trial registration: The trial was registered at the Iranian Registry of Clinical Trials (http://www.irct.ir) with the Irct ID: IRCT2016050527643N2 in the Iranian Registry of Clinical Trials IRCT2015040921670N1.

Funding: The authors received no financial support for the research, authorship, and/or publication of this article. Keywords: Laminaria, Isosorbide dinitrate, Misoprostol, Cervical ripening, Labor, Induced
\end{abstract}

\section{Introduction}

Labor induction before onset of spontaneous uterine contractions is usually performed where risks of pregnancy continuation is high for fetus and/or mother (1). Induction of labor is one of the most common obstetrical interventions. In 2011, labor induction in the United States was performed in $23.2 \%$ of pregnancies while the corresponding figure in 1990 was $9.5 \%$ (2). One of the important factors in labor induction success is cervical

\section{Corresponding author:}

Fateme Borzoee, Department of Operating Room, School of Paramedics, Sabzevar University of Medical Sciences, Sabzevar, Iran. Tel: +98.9358797640, Fax:+98.5144018384, Email: borzoee75026@yahoo.com

Received: July 27, 2017, Accepted: January 22, 2018, Published: May 2018

iThenticate screening: January 04, 2018, English editing: January 24, 2018, Quality control: March 17, 2018

This article has been reviewed / commented by five experts

(C) 2018 The Authors. This is an open access article under the terms of the Creative Commons Attribution-NonCommercialNoDerivs License, which permits use and distribution in any medium, provided the original work is properly cited, the use is non-commercial and no modifications or adaptations are made. 
ripening. A review showed that cesarean rate was lower in women whose cervixes were ripened before induction than women who were directly induced with oxytocin. Labor induction with unripe cervix may also result in induction failure (3), prolonged labor, and instrumental delivery (4). Cervical ripening was usually assessed by Bishop Score. The Bishop Score system is a valid and reliable quantitative method to predict labor induction outcome. Results of a study to compare Bishop Score with other methods for assessing pre-induction cervical ripening, showed that other methods such as transvaginal ultrasound or vaginal fetal fibronectin, did not demonstrate superiority over the Bishop Score method (5). There are different mechanical and cervical methods for preparing an unripe cervix. Selecting a method depends on its safety and efficacy (6). Since 1980, misoprostol, a PGE1 synthetic analogue, has been used to ripen the cervix (7). In a study by Vahid Roudsari, vaginal misoprostol was superior to Foley catheter in terms of delivery time and cesarean rate (8). However, misoprostol may increase the risk of hyper stimulation, caesarean, neonatal intensive care unit (NICU) admission, and meconium passage especially in $50 \mathrm{mg}$ dose (9). Because most patients experience uterine contractions after the initial dose of misoprostol, it is usually considered a labor induction medication rather than a cervical ripener (3). Bearing in mind that an ideal medication must ripen the cervix without stimulating uterine activity, different medicine and methods were examined for cervical ripening. Isosorbide dinitrate (ISDN) and isosorbide mononitrate (ISMN) are vasodilators which produce nitric oxide. They were introduced as a relatively effective and safe method for pre-induction cervical ripening both orally and vaginally, without inducing uterine contraction (10-12). Another benefit of nitric oxide is that it can be used in outpatient wards (13). Results of a systematic review showed that nitric oxide donors can be useful in the process of cervical ripening in comparison to placebo; however, further research is needed to examine the impact of nitric oxide donors on labor duration and delivery outcomes (14). Certain mechanical methods such as laminaria tent which is derived from Laminaria digitata, a large brown alga, may expand the cervix by absorbing water and gradually swelling. Laminaria tents have been used for cervical ripening especially during the second trimester of pregnancy (3). In comparison with vaginal misoprostol, laminaria tent could facilitate hysteroscopic surgery better (15). Regarding the role of cervical preparation on success of labor induction, it is important to find best methods with low side effects for cervical preparation in term pregnancies. To date, studies on the effects of laminaria in term pregnancies have been limited (16). In addition, most studies were conducted to compare misoprostol and ISDN in term pregnancies. The aim of this study is to compare the effects of misoprostol, laminaria, and ISDN on cervical preparation before medical induction of labor.

\section{Material and Methods}

\subsection{Trial design, setting and participants}

This was a three-armed double-blind randomized clinical trial with parallel design, and was conducted at Shahidan Mombini Teaching Hospital of Sabzevar, Iran, from June 2016 to Feb 2017. Shahidan Mombini Hospital is the only hospital in Sabzevar, Iran, and it is affiliated with Sabzevar University of Medical Sciences. This hospital is one of the referral centers of Khorasan Razavi state, Iran. It includes obstetrics and gynecology care with an Obstetrics and Gynecology clinic. Term pregnant women (women with gestational age of 37> weeks) who were hospitalized for induction of labor were recruited. The gestational age was calculated according to the date of the last menstrual period (LMP) with ultrasound confirmation.

\subsection{Selection criteria}

\subsubsection{Inclusion criteria:}

Ninety-six healthy women were selected among 108 women, to participate in the study (Figure 1). The inclusion criteria were: 1) term singleton pregnancies with an indication for labor induction, 2) Bishop Score $<6,3$ ) cephalic presentation, 4) intact water bags, and 5) lack of any contraindication for vaginal delivery.

\subsubsection{Exclusion criteria}

Women with chronic diseases, vaginal bleeding, and those who had used any herbs orally or vaginally to induce labor were excluded from the study. Three women who had perforated water bags and 9 women who had Bishop Score $>6$ were excluded from the study before randomization.

\subsection{Questionnaires}

A demographic questionnaire that includes information such as age, parity, history of abortion, and body mass index was completed first. Another questionnaire including labor progress and delivery of infant was completed during labor and after delivery. Several questions such as dilatation and effacement of the cervix, fetal head station, Bishop Score estimation, oxytocin usage, status of water bags, side effects of medicines, infant birth weight, Apgar score, and amniotic fluid transparency were collected. 


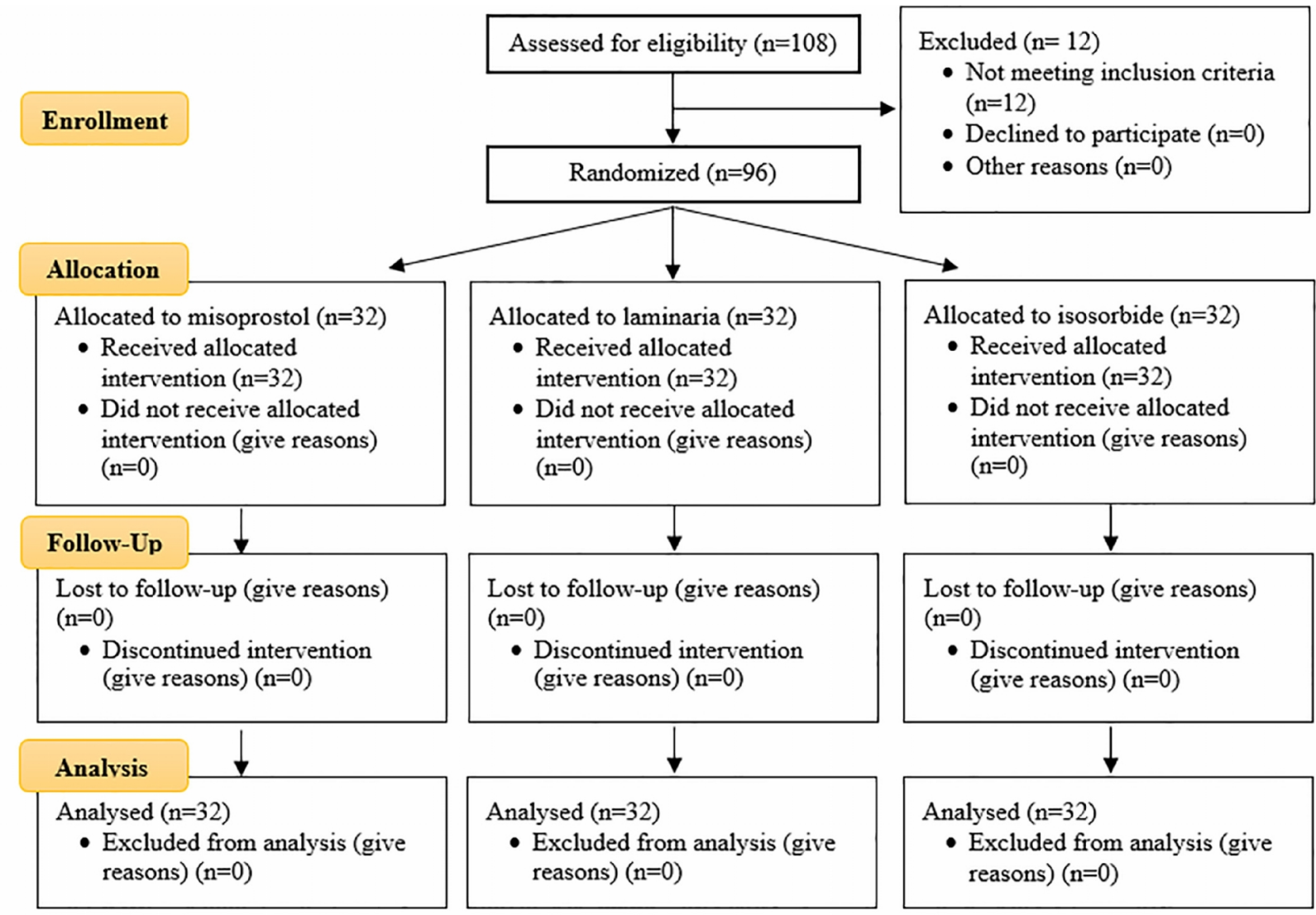

Figure 1. CONSORT diagram of the study

\subsection{Interventions}

In this clinical trial, 96 pregnant women who met the study criteria were recruited. Twelve women were excluded because they met at least one of the exclusion criteria. After knowing the aims of the study, consenting to participate in the study, and completing a demographic questionnaire, the women were allocated into three intervention groups for cervical preparation. In the misoprostol group, $25 \mathrm{mcg}$ misoprostol was inserted into the posterior vaginal fornix. After 6 hours, the same dose of misoprostol was repeated if Bishop Score was lower than 8 and uterine contractions did not start. In the ISDN group, two 20 -mg tablets were inserted into the posterior vaginal fornix. The medication was repeated two times, if after 4 hours Bishop Score was lower than 8 and uterine contractions did not start. In the laminaria group, after washing the vagina with an antiseptic solution and dilating it with a speculum, laminaria was inserted into the cervix with the help of a tenaculum. After 12 hours, the laminaria was removed if it was not expelled spontaneously. In cases of laminaria expulsion or after 12 hours, women were examined if they needed labor induction with oxytocin or the contractions had started. Induction with low dose oxytocin regime was considered when uterine contractions were absent after 12 hours. Similar approach was adopted after inserting 2 doses of ISDN and misoprostol. Cervical ripening was assessed by Bishop Score which estimated through vaginal examination. It is the most commonly used pre-induction scoring system (3). Bishop Score is calculated based on five parameters as follows, cervical dilation, effacement, position, and consistency and fetal station. The maximum score is 13 . A score of 5 or less suggests that labor is unlikely to start spontaneously and induction is unlikely to be successful. Higher Bishop Score denotes better induction outcome (2). Maternal vital signs were taken every 2 hours and monitoring for the evaluation of both uterine contraction and fetal heart rate was performed.

\subsection{Outcomes}

The primary outcomes of our study were Bishop Score improvement in a given time, time to active phase initiation, time to delivery in the three groups, and cesarean rate. The secondary outcomes were the adverse effects of the drugs during the course of the treatments, infants' outcomes including birth weight, Apgar scores of the first and fifth minutes, and length of stay in NICU. 


\subsection{Sample size}

The sample size was calculated to be 32 subjects. This sample size was calculated based on the results of previous study (17) in which the mean and SD of Bishop score after 8 hours in misoprostol and ISDN groups were 5.6 \pm 0.5 and $5.3 \pm 0.4$ and by assuming the test power of $80 \%$ and a confidence level of $95 \%$. No study compared laminaria and misoprostol or ISDN in term pregnancies; so we had to apply the calculated sample size to the third group.

\subsection{Randomization and blinding}

The method of randomization was covariate adaptive randomization in which a new participant is consecutively assigned to a particular treatment group by considering the previous participants' assignments. The first participant was randomly assigned to one of the three treatments and the second participant was randomly assigned to one of the two remaining treatments (18). For blinding, a gynecologist inserted laminaria into the cervical canal, and put misoprostol or isosorbide into the posterior fornix. Three midwives who were unaware of the treatment performed vaginal examinations. In addition, women were not informed which treatment they received. To prevent information bias in estimating the Bishop Scores, midwives' vaginal examinations were matched with each other before the study began.

\subsection{Statistical methods}

Data were analyzed using SPSS version 18 (SPSS Inc., Chicago, Illinois, USA). After normality assessment with one sample K-S test, Kruskal-Wallis and ANOVA tests were used to compare three methods regarding Bishop Score improvement, time to active phase initiation, time to delivery, and Apgar score. Bonferroni test was used to reveal significant differences in mentioned variables between each two groups. The rate of oxytocin administration and mode of delivery in the three groups were compared with chi-square tests.

\subsection{Research ethics}

The ethics committee of Sabzevar University of Medical Sciences approved the study proposal (IR.MEDSAB.REC.1394.3). All women who voluntarily participated in the study and signed a written consent form were subjected to randomization. They were informed that after agreeing o participate in the study they had the right to discontinue with the study whenever they wished without obligation to justify their decisions. There is no routine treatment for unripe cervix and obstetricians usually use misoprostol because of its availability. All medicines were provided by the researchers to the patients, without charge. In case of any significant side effects, the treatment was stopped and the patient was excluded from the study. Moreover, researchers were responsible for any further physical, psychological, and financial expenses.

\section{Results}

Comparisons of the women's characteristics in the three groups are presented in Table 1. The three groups were not different in maternal height, weight, gestational age, parity, maternal age, history of abortion, and Bishop Score before the interventions. Table 2 shows the effects of three interventions on cervical Bishop Score, time between preparation to active phase, time between preparation to delivery, time between induction with oxytocin to delivery, frequency of induction with oxytocin, and cesarean rate. There were significant differences between groups in all mentioned variables. Two-group comparisons using the Tukey HSD test revealed that means of time to active phase $(p<0.001)$, time to delivery $(p<0.001)$, and time from induction with oxytocin to delivery were significantly lower in the misoprostol group than the isosorbide group ( $\mathrm{p}=0.033$ ). In addition, means of time to active phase $(\mathrm{p}<0.001)$ and time to delivery $(\mathrm{p}<0.001)$ were significantly lower in the misoprostol group than the laminaria groups. Time from induction with oxytocin to delivery $(\mathrm{p}=0.056)$ were not different between the misoprostol group and the laminaria group. Means of Bishop Scores changes were higher in the misoprostol group than in the isosorbide groups $(p=0.005)$. Means of Bishop Scores changes were not different in the misoprostol group than the laminaria groups $(p=0.078)$. No difference was observed between laminaria and ISDN in all comparisons. Results indicated that frequency of cesarean was different in the three groups $(\mathrm{p}=0.016)$. In the laminaria group, reasons for cesarean were failure to progress ( 2 cases) and fetal distress accompanied with meconium-stained liquor (3 cases). In the misoprostol group, reasons for cesarean were fetal distress accompanied with meconium-stained liquor (2 cases), uterine hyper tonicity ( 1 cases), and failure to progress (4 cases). Comparison of infant Apgar in the three groups is shown in Table3. First-minute Apgar score was not different in the three groups $(\mathrm{p}=0.362)$, but it was significantly different after 5 minutes $(\mathrm{p}=0.002)$. Tukey HSD test revealed that the mean of five-minute Apgar score was significantly lower in the misoprostol group than the two other groups $(\mathrm{p}=0.007)$. No infants were hospitalized in NICU. Meconium-stained liquor was observed in five cases of the misoprostol group, four cases of the laminaria group, and two cases of the isosorbide group. 
Table 1. Comparisons of women's characteristics in three groups

\begin{tabular}{|c|c|c|c|c|c|}
\hline \multicolumn{2}{|l|}{ Variables } & Laminaria & Misoprostol & ISDN & $\mathrm{p}$-value \\
\hline \multicolumn{2}{|l|}{ Age (year) $(\mathrm{M} \pm \mathrm{SD})$} & $26.18 \pm 4.74$ & $26.62 \pm 5.06$ & $26.59 \pm 4.03$ & 0.915 \\
\hline \multicolumn{2}{|c|}{ Gestational age (week) $(\mathrm{M} \pm \mathrm{SD}) \dagger$} & $40.2 \pm .95$ & $40.6 \pm .66$ & $40.6 \pm .80$ & 0.145 \\
\hline \multicolumn{2}{|c|}{ Birth weight (gram) } & $3415 \pm 329$ & $3280 \pm 330$ & $3459 \pm 378$ & 0.102 \\
\hline \multirow[t]{2}{*}{ Gravidity; n (\%)£ } & Primigravida & $18(56.3)$ & $14(44.8)$ & $7(21.9)$ & \multirow[t]{2}{*}{$0.018^{*}$} \\
\hline & Multigravida & $14(43.7)$ & $18(56.3)$ & $24(75)$ & \\
\hline \multirow[t]{2}{*}{ Parity; n (\%)£ } & Primiparity & $21(65.6)$ & $21(65.6)$ & $16(50.0)$ & \multirow[t]{2}{*}{0.337} \\
\hline & Multipara & $11(34.3)$ & $11(34.3)$ & $16(50)$ & \\
\hline \multirow[t]{2}{*}{ Previous Abortion; n (\%)£ } & Yes & $7(21.9)$ & $11(34.4)$ & $14(43.8)$ & \multirow[t]{2}{*}{0.177} \\
\hline & No & $25(78.1)$ & $21(65.6)$ & $18(56.3)$ & \\
\hline \multicolumn{2}{|l|}{$\mathrm{BMI} \mathrm{Kg} / \mathrm{m}^{2}(\mathrm{M} \pm \mathrm{SD}) \dagger$} & $22.82 \pm 2.33$ & $22.51 \pm 1.60$ & $23.70 \pm 2.00$ & 0.531 \\
\hline \multicolumn{2}{|c|}{ Bishop Score before interventions $(\mathrm{M} \pm \mathrm{SD})$} & $1.94 \pm 1.52$ & $2.5 \pm 1.42$ & $1.97 \pm .93$ & 0.136 \\
\hline
\end{tabular}

$\dagger$ ANOVA, $\ddagger$ Kruskal-Wallis test, $£$ Chi-square test, Isosorbide dinitrate

Table 2. Comparisons of labor and delivery outcomes in three groups

\begin{tabular}{|c|c|c|c|c|c|}
\hline \multicolumn{2}{|l|}{ Variables } & Laminaria & Misoprostol & ISDN & $\mathrm{p}$-value \\
\hline \multicolumn{2}{|l|}{ Bishop Scores changes $(\mathrm{M} \pm \mathrm{SD})+$} & $5.07 \pm 2.71$ & $6.83 \pm 2.73$ & $4.21 \pm 2.6$ & $0.007 *$ \\
\hline \multicolumn{2}{|l|}{ Time to active phase (hours) $(\mathrm{M} \pm \mathrm{SD}) \ddagger$} & $11.25 \pm 3.07$ & $6.22 \pm 3.11$ & $10.12 \pm 3.48$ & $<0.001 * * *$ \\
\hline \multicolumn{2}{|l|}{ Time to delivery (hours) $(\mathrm{M} \pm \mathrm{SD}) \ddagger$} & $17.62 \pm 4.07$ & $11.78 \pm 5.3$ & $17.37 \pm 4.79$ & $<0.001 * * *$ \\
\hline \multirow{2}{*}{$\begin{array}{l}\text { Indication of induction with oxytocin; } n \\
(\%) \dagger\end{array}$} & Yes & $18(56.3)$ & $14(43.8)$ & $30(93.8)$ & \multirow[t]{2}{*}{$<0.001^{* * *}$} \\
\hline & No & $14(43.7)$ & $18(56.3)$ & $12(37.5)$ & \\
\hline \multicolumn{2}{|c|}{$\begin{array}{l}\text { Time from induction with oxytocin to delivery (hour) } \\
(\mathrm{M} \pm \mathrm{SD}) \ddagger\end{array}$} & $8.65 \pm 3.07$ & $5.70 \pm 2.46$ & $8.64 \pm 3.11$ & $0.028 *$ \\
\hline \multirow[t]{2}{*}{ Mode of delivery; $\mathrm{n}(\%) \dagger$} & Cesarean & $5(15.6)$ & $7(21.9)$ & $0(0)$ & \multirow[t]{2}{*}{$0.016^{*}$} \\
\hline & $\begin{array}{l}\text { Vaginal } \\
\text { delivery }\end{array}$ & $27(84.4)$ & $25(78.1)$ & $32(100)$ & \\
\hline \multicolumn{2}{|l|}{ Birth weight $>3500$ grt } & $17(53.1)$ & $10(31.3)$ & $18(56.3)$ & 0.092 \\
\hline \multicolumn{2}{|l|}{ Incidence of side effects; $\mathrm{n}(\%) \dagger$} & 0 & $2(6.3)$ & $19(59.4)$ & $<0.001 * * *$ \\
\hline \multicolumn{2}{|l|}{ Headache (n) } & 0 & 0 & 12 & - \\
\hline \multicolumn{2}{|l|}{ Dizziness (n) } & 0 & 0 & 2 & - \\
\hline \multirow{2}{*}{\multicolumn{2}{|c|}{$\begin{array}{l}\text { Tachycardia (n) } \\
\text { Hyper stimulation (n) }\end{array}$}} & 0 & 0 & 5 & - \\
\hline & & 0 & 2 & 0 & - \\
\hline
\end{tabular}

† Kruskal-Wallis test, $†$ Chi-square test, Isosorbide dinitrate

Table 3. Effects of interventions on Apgar score and meconium -stained Liquor

\begin{tabular}{|l|l|l|l|l|l|}
\hline Variables & Laminaria & Misoprostol & ISDN & $\mathrm{P}$ \\
\hline First minute Apgar $(\mathrm{M} \pm \mathrm{SD}) \ddagger$ & & $8.65 \pm .65$ & $8.75 \pm .67$ & $8.87 \pm .34$ & 0.362 \\
\hline \multicolumn{2}{|l|}{ Five minute Apgar $(\mathrm{M} \pm \mathrm{SD}) \ddagger$} & 10.00 & $9.78 \pm .49$ & 10.00 & $0.002 * *$ \\
\hline Meconium-stained Liquor; $\mathrm{n}(\%) \dagger$ & Yes & $4(12.5)$ & $5(15.6)$ & $2(6.2)$ & \multirow{2}{*}{0.613} \\
\cline { 2 - 5 } & No & $28(87.5)$ & $27(84.4)$ & $30(93.8)$ & \\
\hline
\end{tabular}

$\uparrow$ Kruskal-Wallis test, $†$ Chi-square test, Isosorbide dinitrate

\section{Discussion}

There are different methods to prepare the cervix for induction in the first and second trimester of pregnancy, however, no method has been found to be the best for cervical ripening in term pregnancies. This study was conducted to compare the effects of misoprostol and two other available methods on cervical ripening, labor duration, applying oxytocin, mode of delivery, and Apgar score. Results showed that the three groups were not different regarding confounding and background variables except primigravidity; however, no study demonstrated that having a history of spontaneous or induced abortion in primigravidas may affect cervical ripening process. Bishop Score changes after 12 hours were higher in the misoprostol group than the ISDN and laminaria groups. The lowest change was observed in the ISDN group. Guha's study on both primi- and multiparas with administration of $50 \mathrm{mcg}$ misoprostol and $40 \mathrm{mg}$ MSD every 6 hours, found that changes in cervical score were higher in the misoprostol group than in the IMN group (19). Chanrachakul's study also found that cervical ripening with IMN 
was less effective than misoprostol (18). Results were not in agreement with Haghighi's study which found no difference in Bishop Score changes after 8 hours between ISDN and Misoprostol groups (17). The difference between the two studies may be due to the fact that participants in Haghighi's study were all nulliparous, who have lower response to induction than multiparas, while in our study, $40 \%$ of women were multiparous. Guha's study was also conducted on both primi- and multiparas. Mean time between receiving medication to entering active phase, mean time between inductions to delivery, and mean time between cervical preparations to delivery were shorter in the misoprostol group than the ISDN and laminaria groups while there was no difference between ISDN and laminaria groups. Results were in harmony with previous studies $(17,19,20)$. In Dhillon's study on 50 women, the efficacy and safety of IMN and misoprostol vaginal tablets, applied for cervical ripening prior to suction evacuation of the first trimester abortion were compared. Frequency of doses, induction to ripening interval and intra operative blood loss was significantly higher with the isosorbide mononitrate group as compared to the misoprostol group. Headache and palpitation were the main side effects of IMN. They concluded that misoprostol was a better cervical dilator than IMN in first trimester pregnancy termination with minimal side effects (20). In 62 women, no uterine contraction began 12 hours after medication. Induction with low dose oxytocin regime was started in these cases. The rate of induction with oxytocin was the most in the ISDN group and the least in the misoprostol group. In other words, inserting vaginal misoprostol may increase the chance of spontaneous labor contractions while oxytocin for contraction initiation was indicated in women in the other two groups. In Haghighi's study, labor induction was applied more frequently in the ISDN group than the misoprostol group (17). In Chanrachakul's study, oxytocin was indicated in $92 \%$ of the IMN group and $11 \%$ of the misoprostol group (18). In other words, misoprostol vaginal tablet can ripen the cervix and then initiate labor contractions while ISDN or IMN cannot induce uterine contractions. Despite obtaining good results in the misoprostol group, more cesarean was performed in this group than the laminaria group which is in agreement with previous studies $(8,21)$. That may be due to uterine hyper tonicity caused by misoprostol which result in fetal distress and emergency cesarean. No cesarean was performed in the ISDN group. In Guha's study, cesarean delivery occurred in $23 \%$ in the IMN group and $31 \%$ in the misoprostol group (19). In Khayat's study on 395 women, there were higher cesarean rates and uterine hyperstimulation in the misoprostol group (22.8\%) than Foley catheter combined with vaginal IMN (33.3\%) in term and post-term pregnancies (21). Fortunately, no infant hospitalized in NICU and no difference was observed between groups in terms of meconium-stained amniotic fluid. Although 5-minute Apgar score was lower in the misoprostol group, the difference was not a valuable clinical finding. In Guha's study, fetal side effects were less in the IMN group than the misoprostol group (19). Regarding side effects, women in the laminaria group did not report any side effect while women in the ISDN group experienced the most side effects. In this study, we compared three common and available cervical ripening methods in Iran. Considering the fact that vaginal delivery is the most important outcome and ISDN showed mild unimportant side effects, ISDN was the best method for cervical ripening. We recommend that in future studies combined methods such as laminaria and ISDN/misoprostol be compared to reduce side effects and increase the efficacy of interventions. Our sample consisted of both primi- and multiparas. Since parity may play a role in cervical ripening and response to induction with oxytocin, we recommend that in future studies this variable be considered and sample size be increased to provide comparisons in both primi- and multiparas. One of the limitations of this study was that we did a vaginal examination every 2 hours. It is possible that Bishop Score had changed during the time between two examinations so we could not record it precisely. Another limitation of the study was that there were several psychological variables which may interfere with the improvement of Bishop Score and labor progress that we could not control. The strong point of this study is that we could apply two new methods, and compared three methods in term pregnant women. In addition, laminaria has usually been used in the second trimester of pregnancy while we applied it in term pregnancies. Finally, we achieved similar results with applying low dose misoprostol and ISDN in comparison to previous studies which used a double dose of both medicines.

\section{Conclusions}

Misoprostol is more effective than ISDN and laminaria tent for ripening of cervix before induction, and can decrease labor duration and time to delivery. However, cesarean rate due to fetal distress was higher in the misoprostol group than the laminaria and ISDN groups. In contrast, ISDN had a higher rate of vaginal delivery. Although mild side effects were seen after applying ISDN vaginal tab, those side effects did not intervene with the labor and delivery process, and did not increase cesarean rate. Considering the fact that the most important expected outcome is vaginal delivery rate, ISDN is the best method in our study. We recommend more studies to examine side effects of isosorbide dinitrate on fetus and mothers during early postpartum. Since we applied a low dosage of ISDN, further study on the use of isosorbide dinitrate with higher dosage on primiparas is a good path for the continuing research in this subject. 


\section{Acknowledgments:}

This study was approved by the Research Chancellery of Sabzevar University of Medical Sciences (approval number $=3930102156)$ as a thesis for the fulfillment of medical doctoral degree. We appreciate all the women who participated in this study.

\section{Trial Registration:}

The trial was registered at the Iranian Registry of Clinical Trials (http://www.irct.ir) with the Irct ID: IRCT2015040921670N1.

\section{Funding:}

The authors received no financial support for the research, authorship, and/or publication of this article.

\section{Conflict of Interest:}

There is no conflict of interest to be declared.

\section{Authors' contributions:}

All authors contributed to this project and article equally. All authors read and approved the final manuscript.

\section{References:}

1) James D, Steet P, Weiner C. High risk pregnancy management options. 6th ed. Philadelphia: Saunders Co; 2007.

2) Fox KA, Ramirez MM, Ramin SM. Induction and augmentation of labor. Management of Labor and Delivery: John Wiley \& Sons, Ltd; 2015: 62-85. doi: 10.1002/9781118327241.ch4.

3) Sanchez-Ramos L. Induction of labor. Obstet Gynecol Clin North Am. 2005; 32: 181-200. doi: 10.1016/j.ogc.2004.12.004.

4) Hofmeyr GJ, Gulmezoglu AM, Pileggi C. Vaginal misoprostol for cervical ripening and induction of labour. Cochrane Database Syst Rev. 2010; (10): CD000941. doi: 10.1002/14651858.CD000941.pub2. PMID: 20927722.

5) Ezebialu IU, Eke AC, Eleje GU, Nwachukwu CE. Methods for assessing pre-induction cervical ripening. Cochrane Database Syst Rev. 2015. doi: 10.1002/14651858.CD010762.pub2.

6) Alfirevic Z, Aflaifel N, Weeks A. Oral misoprostol for induction of labour. Cochrane Database Syst Rev. 2014; (6): CD001338. doi: 10.1002/14651858.CD001338.pub3. PMID: 24924489.

7) Cunnigham F, Leveno K, Bloom S, Haulth J, Rouse D, Spong C. Williams obstetrics. 23rd ed. New York: McGraw Hill; 2010.

8) Vahid Roudsari F, Ayati S, Ghasemi M, Hasanzadeh Mofrad M, Shakeri MT, Farshidi F, et al. Comparison of vaginal misoprostol with foley catheter for cervical ripening and induction of labor. Iran J Pharm Res. 2011; 10: 149-54.

9) Stephenson ML, Wing DA. A novel misoprostol delivery system for induction of labor: clinical utility and patient considerations. Drug Des Devel Ther. 2015; 9: 2321-7. doi: 10.2147/dddt.s64227.

10) Osman I, MacKenzie F, Norrie J, Murray HM, Greer IA, Norman JE. The "PRIM" study: a randomized comparison of prostaglandin E2 gel with the nitric oxide donor isosorbide mononitrate for cervical ripening before the induction of labor at term. Am J Obstet Gynecol. 2006; 194: 1012-21. doi: 10.1016/j.ajog.2005.10.812.

11) Haghighi L, Moukhah S, Goshtasbi A. Comparing the effect of oral and vaginal isosorbide dinitrate in preinduction cervical ripening in term pregnancy: A controlled clinical trial. Advanced Biomedical Research 2015; 4: 129. doi: 10.4103/2277-9175.158259.

12) Yazdizadeh H, Abedi P, Najar S, Angali KA. The impact of isosorbide mononitrate on cervical ripening and labor induction in primiparous women with term pregnancy: A double-blind, randomized, controlled trial. Iranian Journal of Nursing and Midwifery Research. 2013; 18: 246-50. PMID: 23983763, PMCID: PMC3748546.

13) Amorosa JM, Stone JL. Outpatient cervical ripening. Semin Perinatol. 2015; 39: 488-94. doi: 10.1053/j.semperi.2015.07.014.

14) Ghosh A, Lattey KR, Kelly AJ. Nitric oxide donors for cervical ripening and induction of labour. Cochrane Database Syst Rev. 2016; 12: CD006901. doi: 10.1002/14651858.CD006901.pub3. PMID: 27918616.

15) Karakus S, Akkar OB, Yildiz C, Yenicesu GI, Cetin M, Cetin A. Comparison of Effectiveness of Laminaria versus Vaginal Misoprostol for Cervical Preparation Before Operative Hysteroscopy in Women 
of Reproductive Age: A Prospective Randomized Trial. J Minim Invasive Gynecol. 2016; 23: 46-52. doi: 10.1016/j.jmig.2015.08.006.

16) Jozwiak M, Bloemenkamp KW, Kelly AJ, Mol BW, Irion O, Boulvain M. Mechanical methods for induction of labour. Cochrane Database Syst Rev. 2012; (3): CD001233. doi: 10.1002/14651858.CD001233.pub2. PMID: 22419277.

17) Haghighi L, Homam H, Raoofi $Z$, Najmi Z. Intravaginal isosorbide dinitrate or misoprostol for cervical ripening prior to induction of labour: a randomised controlled trial. J Obstet Gynaecol. 2013; 33: 272-6. doi: 10.3109/01443615.2012.753422.

18) Chanrachakul B, Herabutya Y, Punyavachira P. Randomized trial of isosorbide mononitrate versus misoprostol for cervical ripening at term. Int J Gynaecol Obstet. 2002; 78: 139-45. doi: 10.1016/S00207292(02)00128-5.

19) Guha K, Fatema A, Biswas PK, Haque E. Isosorbide Mononitrate versus Misoprostol for Cervical Ripening and Induction of Labour at Term. Mymensingh Med J. 2015; 24: 346-51.

20) Dhillon APK, Shergill HK, Yadav P. Comparative study between vaginal isosorbide mononitrate and misoprostol for induction of cervical ripening prior to surgical evacuation of first trimester embryonic demise. Int J Reprod Contracept Obstet Gynecol. 2015; 4: 398-401. doi: 10.5455/23201770.ijrcog20150422.

21) El-Khayat W, Alelaiw H, El-kateb A, Elsemary A. Comparing vaginal misoprostol versus Foley catheter plus vaginal isosorbide mononitrate for labor induction. J Matern Fetal Neonatal Med. 2016; 29: 487-92. doi: 10.3109/14767058.2015.1007036. 